\title{
Designing Scalable WDM Optical Interconnects Using Predefined Wavelength Conversion
}

\author{
Haitham S. Hamza and Jitender S. Deogun \\ Department of Computer Science \& Engineering, \\ University of Nebraska-Lincoln, \\ Lincoln, NE 68588-0115, USA \\ \{hhamza, deogun\}@cse.unl.edu
}

\begin{abstract}
This paper investigates the problem of designing scalable and cost-effective wavelength division multiplexing (WDM) optical interconnects. We propose a new design for WDM optical interconnect that has several advantages over existing designs. First, wavelength conversion occurs between two predefined wavelengths. This not only eliminates the need for expensive wide-range wavelength converters, but also ensures high scalability as the conversion range is independent of the number of the wavelengths in the system. Second, the new design requires a smaller number of switching elements compared to most of the recent best interconnect designs.
\end{abstract}

\section{Introduction}

A Wavelength Division Multiplexing (WDM) interconnect provides the basic functionality of switching a signal arriving at an input fiber on one of the possible wavelengths to an output fiber on a possibly different wavelength, while maintaining the signal in the optical domain. In the absence of Wavelength Converters (WCs); an optical interconnect can only switch signals in the "space domain": an input signal on a given wavelength can be connected to any output fiber without changing its wavelength (given that this wavelength is free on the required output fiber). Switching only in the space domain, however, limits the capability of the interconnect. Thus, introduction of switching in both space and wavelength domains enhances the capability of interconnects. Several interconnect with space and wavelength switching capabilities have been proposed over the last few years, e.g. [11, 17, 38, 39.

However, as WDM enables more and more wavelengths per fiber, the design of cost-effective and scalable WDM optical interconnects becomes a real challenge as the number of required switches and WCs increases. Large number of switches and WCs can lead to architectures that are impractical or economically infeasible. In general, the cost of a WDM interconnect is dominated by two factors: the switching cost and the wavelength conversion cost [11] 38] [39]. Switching cost is proportional to the total number of switching elements (SEs) in the interconnect 38] 39]. 
Conversion cost, on the other hand, depends on the total number of WCs as well as the conversion cost of each WC. Let $\Lambda$ be the set of wavelengths in the network, and let $S$ and $D$ be two sets of wavelengths such that $S \subseteq \Lambda$ and $D \subseteq \Lambda$. Denote by $\mathrm{WC}(S, D)$ a WC that is capable of converting any wavelength in set $S$ to any wavelength in set $D$. The conversion cost of $\mathrm{WC}(S, D)$ is therefore proportional to $|S| .|D|, 1 \leq|S|,|D| \leq|\Lambda|[38$. Obviously, the cheapest WC is the one with $|S|=|D|=1$, or a Fixed-range WC. Most existing WDM interconnects, however, make use of Full-range WCs (FWC) where $|S|=|D|=W[11] 39$. Typical WDM interconnects are large, and for these FWCs can be very expensive and difficult to implement and thus, more recent interconnects adapt Limitedrange WCs (LWCs) instead of FWCs 38.

Using LWCs, however, may not lead to cost-effective and scalable WDM design, particularly for large interconnects. This because, the conversion range of LWCs is proportional to the number wavelengths in the system. Therefore, as the number of wavelengths in the system increases, so does the conversion range of LWCs. We argue that, in order to develop cost-effective and highly scalable WDM interconnects, the range of used wavelength conversion must be independent of the number of wavelengths in the system. Accordingly, in this paper, we investigate a new design for WDM optical interconnects that require "only" wavelength conversion between two predefined wavelengths while providing a full-connectivity between input and output fibers. The new design exploits the potential of the Wavelength Exchange Optical Crossbar (WOC) - a device that can switch signals simultaneously and seamlessly both in space and wavelength domains [17. The proposed design follows the recursive structure of the wellknown Clos network [5], and hence, scalability occurs in an orderly fashion.

The reminder of the paper is organized as following. Section 2 provides an overview of existing WDM optical interconnect design approaches. The proposed design is presented in Section 3. Section 4 investigates the hardware complexity of the new design. A comparison of different interconnect designs is given in Section 5; Conclusions are presented in Section 6.

\section{Existing Designs for WDM Optical Interconnect}

Several WDM interconnect designs have been investigated in the literature, e.g. [1, [3], 9], 11, [13]-[16, 20], 26], 27], 36]-38. A generic $F W \times F W$ WDM optical interconnect (where $F$ and $W$ represent, respectively, the number of fibers and wavelengths per fiber) consists of two main modules: a space switching unit (a switching unit, for short) and one or more wavelength conversion units (conversion units). In the following, we classify existing interconnect architectures based on the design of their switching and conversion units.

- Design of Switching Units. Switching units can be broadly classified into two main categorizes: Single Stage and Multistage designs:

1. Single Stage: In this design, a single $F W \times F W$ switching fabric is used [37, 38. Such a design requires $F^{2} W^{2}$ switching elements which can be 
very expensive for a typical large-scale WDM interconnect with hundreds of fibers and wavelengths.

2. Multistage: Multistage Interconnection Networks (MINs) are used to economically realize large-scale interconnects 32 . A MIN interconnects a set of input ports to a set of outputs ports using several stages of fixedsize switching modules. Electronic MINs have been extensively investigated in the literature and several designs were propagated to the optical domain, e.g. 11, 3], 9], 13], 14, 17], 20], 26], 27], 38], 39].

- Design of Conversion Units. Conversion units are realized using wavelength converters. Regardless of their technology, WC can be generally classified into Full-range or Limited-range converters. Designs for conversion units can be classified into three main categorizes:

1. Dedicated Wavelength Conversion: In this approach, each input and/or output port is assigned a dedicated WC. Thus, a $F W \times F W$ interconnect under this design will have at least FW WCs. These WCs can be FWCs, e.g. 11, 37, 39, or LWCs (and SWCs), e.g. [15, [16, 36, 38. With large number of wavelengths, however, this approach can lead to designs that are impractical, especially if FWCs are used.

2. Wavelength Converter Banks: In this design scheme, a pool of WCs is allocated in the interconnect [23, 28. Only signals that require wavelength conversion are directed to this pool and then switched to the required output port. In such a design, there is a trade-off between the number of converter banks and WCs within each bank; the cost and complexity of switching; and the permutation capacity of the interconnect (i.e. the number of permutations patterns that the interconnect can realize).

3. Bulk Wavelength Conversion: This scheme adapts bulk WCs that are capable of converting multiple wavelengths simultaneously. Interconnects that use bulk WCs are known as wave-mixing interconnects [1, 27. Wave-mixing interconnects considerably reduce the number of WCs compared to other design approaches, while avoiding the added complexity of the shared conversion bank design discussed above. However, these designs may introduce up to $O(\log W)$ wavelength conversion stages [27] , and hence, the length of the signal path within the switch increases. These extra stages not only increase hardware complexity but also increase the length of the signal path. As a result, delay, signal attenuation, and accumulated cross-talk noise are also increased [8].

\section{The Proposed WDM Interconnect}

The proposed design is based on the well-known Clos network [5]. An $N \times N$ 3-stage Clos network, denoted as $C(m, n, r)$, has $r$ switches of $n \times m$ size each in the first stage; $m$ switches of $r \times r$ size each in the second; and $r$ switches of $m \times n$ size each in the third stage, and $N=r . n$. The parameter $m$ determines the blocking characteristics of the Clos network. For brevity, we consider only 
rearrangable nonblocking interconnects, i.e. $m=n[22$, and extensions to strictly nonblocking designs follows easily. In the following, we first review the main building blocks of the proposed interconnect and then we present the structure of the new design.

\subsection{Wavelength Exchange Optical Crossbar}

This section briefly reviews the concepts of WOCs and WDM crossbar switches used in the proposed design. A WOC has two input ports, two output ports, and a control signal (Figure 1) 17. The input to a WOC is two signals $S_{1}$ at wavelength $\lambda_{a}$, and $S_{2}$ at wavelength $\lambda_{b}$. When the control signal is $O F F$; an input signal to the WOC appears at an output port with the same wavelength. Conversely, when the control signal is $O N$, the WOC performs both switching and conversion simultaneously. WOC can be realized by simultaneous power exchange between the two input signals, a phenomenon that has been theoretically and experimentally demonstrated using Four Wave Mixing (FWM) 24] 25, and Photonic Crystal [2]. It is worth noting that, a WOC performs predefined fixed wavelength conversion and hence $|S|=|D|=1$.

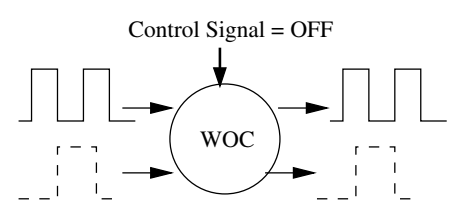

(a)

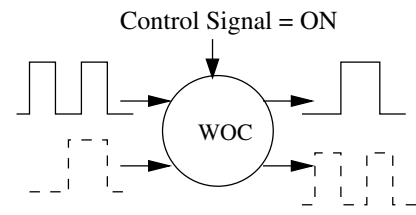

(b)

$$
\lambda_{1} \longrightarrow \lambda_{2}-\cdots--
$$

Fig. 1. The WOC device and its different configurations: (a) Bar state (b) Simultaneous switching and wavelength conversion

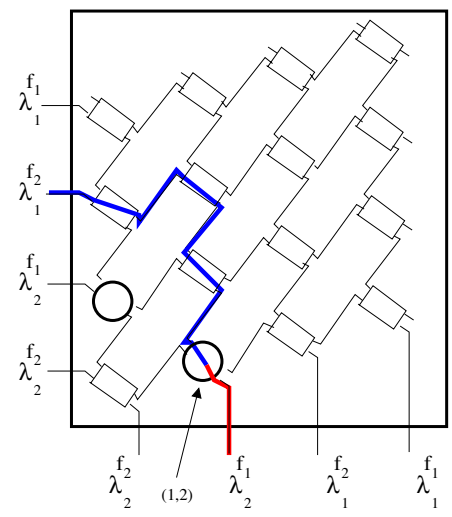

(a)

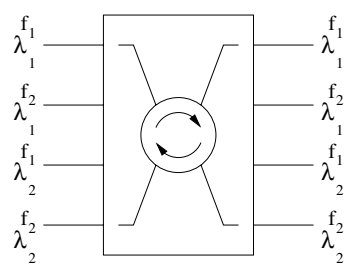

(b)

Fig. 2. (a) The $2^{\lambda}(2 \times 2)$ WDM crossbar switch, (b) Symbolic notation 


\subsection{WDM Crossbar Switches}

Let $W^{\lambda}(F \times F)$ denotes an $N \times N$ WDM interconnect with $F$ input and $F$ output fibers, where each fiber has $W$ wavelengths, and $N=F W$. Without lose of generality, $F$ and $W$ are assumed to be powers of 2 . We denote by $\lambda_{w}^{f_{j}}$, a signal on wavelength $\lambda_{w}$ in fiber $j$. Figure 2 shows a $2^{\lambda}(2 \times 2)$ WDM crossbar and symbolic notation. Unlike conventional crossbars where signals can be switched only in the space domain, WDM crossbars employ WOCs and thus they can switch in both space and wavelength domains. The label $(1,2)$ in the figure indicates a WOC that exchanges signal between $\lambda_{1}$ and $\lambda_{2}$.

\subsection{A New WDM Interconnect Design}

The basic idea in the new design is to perform pure space switching in the first and third stages while any needed wavelength conversion is performed in the middle stage. Therefore, in our design, space crossbars are used in the first and third stages, whereas WDM crossbars in the middle stage. Thus, there are $r$ switches each of size $1^{\lambda}(n \times n)$ in the first stage $(2 \leq n \leq F-1) ; n$ switches each of size $\left(\frac{N}{F}\right)^{\lambda}\left(\frac{F}{n} \times \frac{F}{n}\right)$ in the second stage; and $r$ switches each of size $1^{\lambda}(n \times n)$ in the third stage.

Different values of $n$ lead to different interconnect structures, thus, there are $F-1$ different designs for a $W^{\lambda}(F \times F)$ interconnect. However, if we assume $n=2^{k}, 1 \leq k \leq \log _{2} F$, then there are at most $\log _{2} F$ designs.

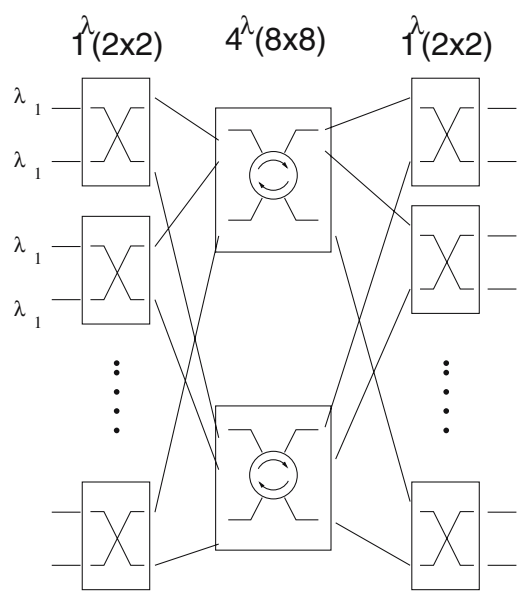

(a)

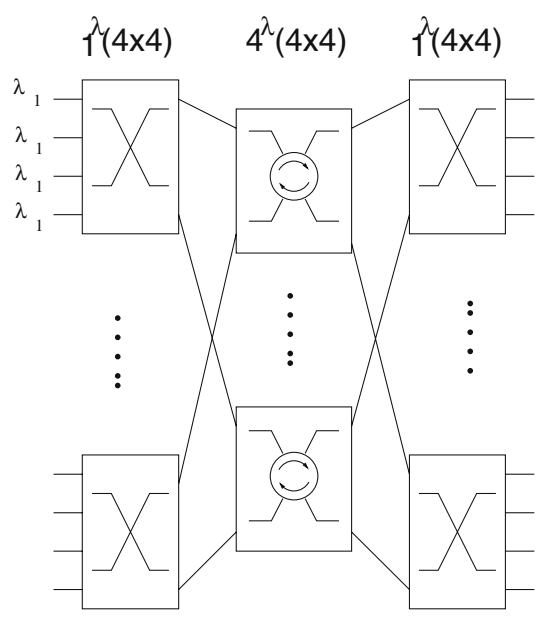

(b)

Fig. 3. Two possible designs of $4^{\lambda}(16 \times 16)$ WDM interconnect with: (a) $n=2$, and (b) $n=4$. 


\subsection{An Example: The $4^{\lambda}(16 \times 16)$ WDM Interconnect}

Here we show a $4^{\lambda}(16 \times 16)$ interconnect using the proposed design approach. Since $F=16$, hence, there are $4\left(=\log _{2} 16\right)$ different designs. The cost of these designs is given in Table 1, It is worth noting that the four designs have the same number of WOCs. As we show later in this paper, the number of WOCs does not depend on the value of $n$. Figure 3 shows two $4^{\lambda}(16 \times 16)$ interconnect designs for $n=2$ and $n=4$.

Table 1. Four different designs and their hardware cost for a $4^{\lambda}(16 \times 16)$ WDM interconnect

\begin{tabular}{|l||l|l|l||l|l|}
\hline$n$ & First & Middle & Third & \# SEs & \# WOCs \\
\hline \hline 2 & $1^{\lambda}(2 \times 2)$ & $4^{\lambda}(8 \times 8)$ & $1^{\lambda}(2 \times 2)$. & 2208 & 96 \\
\hline 4 & $1^{\lambda}(4 \times 4)$ & $4^{\lambda}(4 \times 4)$ & $1^{\lambda}(4 \times 4)$ & 1440 & 96 \\
\hline 8 & $1^{\lambda}(8 \times 8)$ & $4^{\lambda}(2 \times 2)$ & $1^{\lambda}(8 \times 8)$ & 1440 & 96 \\
\hline 16 & $1^{\lambda}(32 \times 32)$ & $4^{\lambda}(1 \times 1)$ & $1^{\lambda}(32 \times 32)$ & 8512 & 96 \\
\hline
\end{tabular}

\section{Hardware Cost}

To estimate the hardware complexity of the new design, we compute the overall number of switches and WOCs. In the following, we compute the cost of a WDM interconnect under the proposed design, and then, we drive the values of $n$ to design an interconnect with minimum hardware cost.

Lemma 1. the total number of SEs and WOCs in a $W^{\lambda}(F \times F)$ WDM crossbar is:

$$
\begin{gathered}
\# S E=\frac{N}{2}(2 N-W+1) \\
\# W O C=\frac{N}{2}(W-1)
\end{gathered}
$$

Proof. The proof follows directly from [18].

Lemma 2. the total number of SEs and WOCs in a $W^{\lambda}(F \times F) W D M$ crossbar is:

$$
\begin{gathered}
\# S E s=2 N n+\frac{N^{2}}{n}-\frac{N}{2}(W-1) \\
\# W O C s=\frac{N}{2}(W-1)
\end{gathered}
$$

Proof. The first and third stages consist of switches of size $n^{2}$, therefore, there are 2.r. $n^{2}$ SEs in these two stages. The number of SEs in the middle stage can 
be computed by substituting $N$ with $(N / n)$ (the size of a WDM crossbar in the middle stage) in Equation (11). Thus, the total number of SEs is:

$$
\# S E s=2 . r . n^{2}+r \cdot \frac{N}{2 n} \cdot\left(\frac{2 N}{n}-\frac{N}{F}+1\right)
$$

Substituting $r$ with $N / n$ in the above equation, we obtain:

$$
\# S E s=2 N n+\frac{N^{2}}{n}-\frac{N}{2}(W-1)
$$

The number of WOCs depends only on the size of the switches in the middle stage. It is straightforward to show that the total number of WOCs in the new design is:

$$
\# W O C s=\frac{N}{2}(W-1)
$$

Since different values of $n$ result in different designs with different hardware costs, it is interesting to investigate the value of $n$ that leads to an interconnect with minimum hardware cost. It may be noted from (7) that the number of WOCs is independent on the value of $n$. Therefore, to optimize the cost of an interconnect under our design, it is sufficient to minimize the number of SEs.

To find the optimal value of $n$ that minimizes the total number of SEs, we set the derivation of \# SEs in Equation (6) to zero, to obtain:

$$
n=\frac{1}{\sqrt{2}} \cdot N^{\frac{1}{2}} .
$$

By substituting the value of $n$ above in the total number of SEs in Equation (6), we obtain:

$$
\# \mathrm{SEs}=(2 N)^{\frac{3}{2}}-\frac{N}{2}(W-1)
$$

\section{Comparison of Designs}

Recent WDM interconnect designs with sparse crossbar switches can potentially reduce the number of SEs and have shown to be very cost-effective compared to existing interconnect designs [38] 39]. Therefore, it is sufficient to compare our design with these two recent designs (See Table 2). The Sparse/FWC interconnect design in 39] requires the minimum number of SEs compared to existing WDM interconnects including the proposed. However, the design in 39] requires $F W$ Full-range WCs $(|S|=|D|=W)$, leading to $O\left(W^{3}\right)$ conversion cost, which makes the design impractical or economically infeasible when the number of wavelengths increases.

To reduce the conversion cost while controlling the number of SEs, the Sparse/LWC interconnect 38] make use of LWCs and sparse crossbar switches. 
Sparse/LWC employs Fixed-range WCs $(|S|=|D|=1)$ at the input of the interconnect to convert all input signals to a specific wavelength. At the output of the interconnect, LWCs with conversion cost $c(|S|=1$ and $|D|=c, 1 \leq c \leq W)$ are used in order to convert output signals to the required wavelength.

Clearly, Sparse/LWC interconnects are more practical as compared to the Sparse/FWC designs, and hence, in the following, we focus on comparing our design with the Sparse/ $L W C$ interconnects. We show that, for the same conversion cost, our design requires a smaller number of SEs compared to the Sparse/LWC design. This can be shown by computing the value of $c$ by equating the conversion cost of both designs:

$$
\begin{aligned}
\frac{N}{2}(W-1) & =N(c+1)-F, \text { thus: } \\
c & =\frac{W-3}{2}+\frac{1}{W}
\end{aligned}
$$

Table 2. A Summary of \# Crosspoints, and Conversion Cost of the different WDM interconnect designs. $(1 \leq c \leq W)$.

\begin{tabular}{|l|l|l|}
\hline Design & \# Crosspoints & Conversion Cost \\
\hline \hline S/FWC [39] & $(2 N)^{3 / 2}-N(W-1)$ & $N W^{2}$ \\
\hline S/LWC [38] & $(2 N)^{3 / 2}-N(c-1)$ & $N(c+1)-F$ \\
\hline New & $(2 N)^{\frac{3}{2}}-\frac{N}{2}(W-1)$ & $\frac{N}{2}(W-1)$ \\
\hline
\end{tabular}

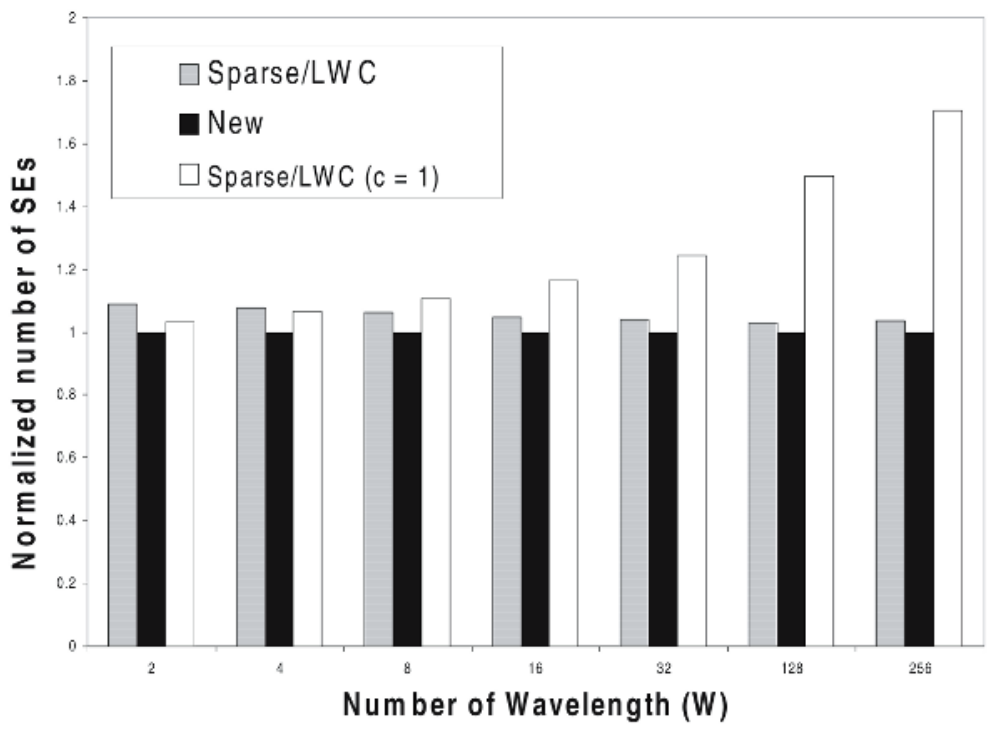

Fig. 4. The normalized number of SEs of new and Sparsedesigns for different values of $W$ and $F=16$ 


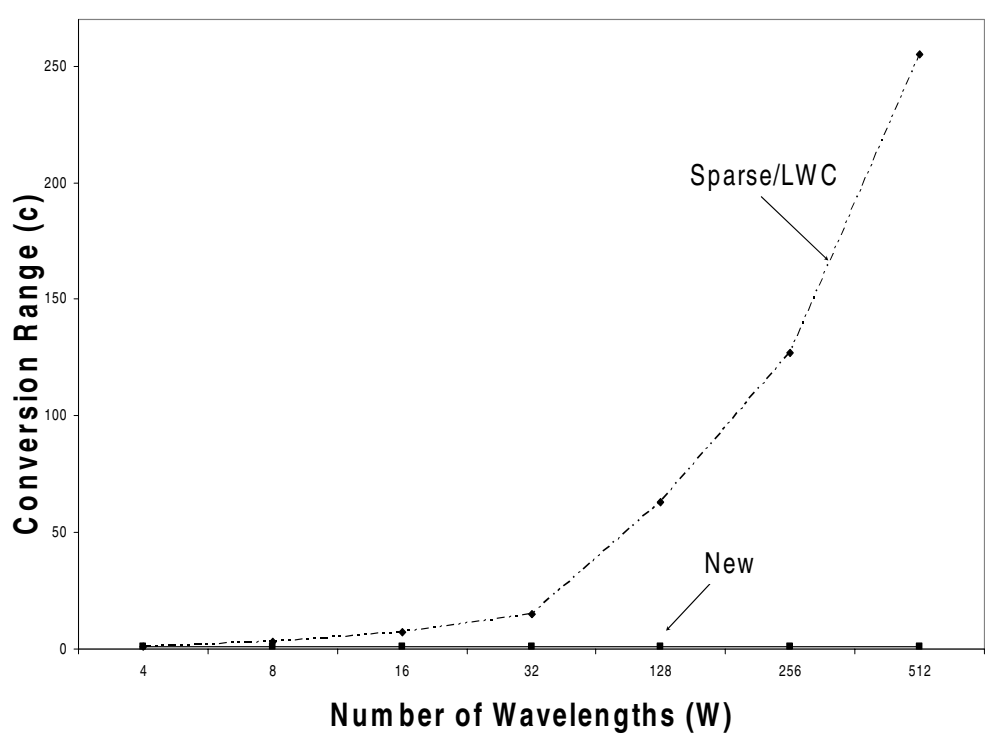

Fig. 5. The value of the conversion-range $c$ as a function of the number of wavelengths $(F=16)$

Using the above value of $c$, Figure 4 shows the total number of SEs for the Sparse/ $L W C$ design normalized to the total number of SEs in our design for an interconnect with $F=16$ and for different number of wavelengths. As shown in the figure, our design has smaller number of SEs compared to the Sparse/LWC design. Also, it may be noted that the conversion range, $c$, for the LWCs in Sparse/LWC design increases as the number of wavelengths increases (See Figure 5) even when both designs have the same overall conversion cost. Moreover, as conversion range increases the WCs become harder to implement with current optical technologies, limiting the scalability of the Sparse/LWC design.

It may be noted that a Sparse/ $L W C$ interconnect can be designed using only fixed-range WCs by selecting $c=1$ [38]. Such designs render smaller conversion cost by increasing the number of SEs (See Figure 4). As the number of wavelengths increases, this design may require more than 1.5 times the number of SEs in our design.

It should be pointed out, however, that, an accurate comparison of different designs should take into consideration the overall cost of an interconnect. Indeed, since the proposed design and the Sparse/LWC are based on two different technologies, thus, the actual costs of WOCs, WCs, and SEs, will determine which of the two designs has a smaller overall hardware cost. For example, if the cost of WOCs is higher than that of WCs, then, the Sparse/LWC design will probably have a smaller overall cost compared to the proposed design. Such analysis is difficult since we do not have a good estimation of the actual cost of WOCs, however, one can analyze a range of values for each of the different components (i.e. WOCs, WCs, and SEs) and identify the regions in which each design will have a smaller cost, we leave this investigation for future work. 
Although, we have focused our comparison on the number of SEs and the conversion cost, it is worth noting that sparse crossbars require complex routing algorithms compared to full crossbars 38. Our design, on the other hand, preserves the structure of full crossbars, and hence, any existing routing algorithm can be readily adapted for our design. Therefore, the new design provides "fast and simple" switching.

\section{Conclusions}

We propose a new WDM optical interconnect design that provides full-connectivity while performing conversion between predefined wavelengths, and hence, eliminating the need for expensive full- and wide-range wavelength converters used in most designs. This not only reduces the conversion cost, but also provides fast and simple switching. Moreover, the conversion range is independent of the number of wavelengths in the network which improves the scalability of interconnect. In addition, for the same conversion cost, the proposed design requires a smaller number of SEs compared to best known designs.

\section{Acknowledgment}

This work was supported, in part, by an NSF EPSCoR grant EPS- 0346476 and by a Nebraska Research Initiative grant. The authors would like to thank the anonymous reviewers for their suggestions for improving this paper.

\section{References}

1. A.C. Dasylva, D.Y. Montuno, and P. Kodaypak, "Nonblocking space-wavelength networks with wave-mixing frequency conversion," J. Opt. Netw. 1, pp. 206-216, 2002.

2. A. Chowdhury, S.C. Hagness, and L. McCaughan, "Simultaneous optical wavelength interchange with a two-dimensional second-order nonlinear photonic crystal," Opt. Lett., vol. 25, No.11, June 2000, pp. 832-834.

3. A. Rasala and G. Wilfong, "Strictly non-blocking WDM cross-connects," In Proc. of the Eleventh Annual ACM-SIAM Symposium on Discrete Algorithms (SODA'02), pp. 606-615, 2000.

4. B. Mukherjee, "WDM optical communication netowrks: progress and challenges," IEEE JSAC., vol. 18 , no. 10 pp. 1810-1824, 2000.

5. C. Clos, "A study of non-blocking switching networks," Bell System Tech. J., pp. 407-424, 1958.

6. C. Qiao and M. Yoo, "Optical burst switching (obs)- a new paradigm for an optical internet," J. of High Speed Networks, vol.8, no.1, pp. 69-84, 1999.

7. D.C. Opferman and N.T. Tsao-Wu, "On a class of rearrangeable switching networks, Part I: control algorithm," Bell Syst. Tech. J., vol. 5, no. 50, pp. 1579-1600, 1971.

8. D. Pan, V. Anand, and H.Q. Ngo, "Cost-effective constructions for nonblocking WDM multicast switching networks," IEEE ICC 04, pp. 1801-1805 
9. F.K. Hwang, "A survey of nonblocking multicast three-stage Clos networks," IEEE Com. Mag., pp. 34-37, 2003.

10. G.R. Hill, et al., "A transport network layer based on optical netowrk elements," J. Lightwave Tech. vol 11, pp. 667-679, May/June 1993.

11. G. Wilfong, B. Mikkelsen, C. Doerr, and M. Zirngibl, "WDM cross-connect architectures with reduced complexity," J. of Lightwave Tech.. vol. 17, no. 10, 1999, pp. $1732-1741$.

12. G. Xiao and Y.W. Leung, "Algorithms for allocating wavelength converters in alloptical networks," IEEE/ACM Trans. on Networking, vol. 7, pp. 545-557, 1999.

13. H. Jonathan Chao, K-L. Deng, and Z. Jing, "PetaStar: A Petabit photonic packet switch," IEEE JSAC., vol. 21, no. 7, pp. 1096-1112, 2003.

14. H. Jonathan Chao, Z. Jing, and S.Y. Liew, "Matching algorithms for three-stage bufferless Clos network switches," IEEE Communication Mag., pp. 46-54, 2003.

15. H.Q. Ngo, D. Pan, and C. Qiao, "Nonblocking WDM switches based on arrayed waveguide grating and limited wavelegnth conversion," Proc. 23rd IEEE INFOCOM 04', 2004.

16. H.Q. Ngo, D. Pan, and Y. Yang, "Optical switching networks with minimum number of limited range wavelength converters," Proc. 24rd IEEE INFOCOM 2005, 2005.

17. H.S. Hamza and J.S. Deogun, "Wavelength exchanging Cross-Connect (WEX)- a new class of photonic cross-connect architectures," IEEE/OSA J. Lightwave Tech., ( To appear).

18. H.S. Hamza and J.S. Deogun, "WDM Optical Interconnects - A Balanced Design Approach," Manuscript under review.

19. H. S. Hinton, "A nonblocking optical interconnection network using directional couplers," GLOBECOM 1984, pp. 26.5.1-26.5.5, 1984.

20. J. Cheyns et al., "Clos lives on in optical packet switching," IEEE Com. Mag., pp. 114-121, 2003.

21. J. Ramamirtham and J.S. Turner, "Design of wavelength converting switches for optical burst switching," in Proc. of the 21st Annual Joint Conference of the IEEE Computer and Communications Societies (INFOCOM 02'), vol. 2, pp. 1162-1171, 2005.

22. J.Y. Hui, Switching and traffic theory for integrated broadband network, "Pointto-point multistage circuit switching," Kluwer, pp. 53-83, 1990.

23. K.-C. Lee and V.O.K. Li, "A wavelength-convertible optical network," J. of Lightwave Tech., vol. 11, pp. 962-970, 1993.

24. K. Moei, H. Takara and M. Saruwatari, "Wavelength interchange with an optical parametric loop mirror," Electronics Lett., vol.33, no.6, pp. 520 -522, Mar 1997.

25. K. Uesaka, K. K-Y. Wong, M.E. Marhic, and L.G. Kazovsky, "Wavelength Exchange in a Highly Nonlinear Dispersion-Shifted Fiber: Theory and Experiments," IEEE J. of Selected Topics in Quantum Electronics, vol. 8, no. 3, pp. 560-568, May/June 2002.

26. K. Zhu, H. Zang, and B. Mukherjee "A comprehensive study on next-generation optical grooming switches," IEEE JSAC., vol. 21, no. 7, pp. 1173-1186, 2003.

27. N. Antoniades, S.J.B. Yoo, K. Bala, G. Ellinas, and T.E. Stern, "An architecture for a wavelength-Interchanging cross-connect utilizing parametric wavelength converters," J. of Lightwave Tech., vol. 17. no. 7, July 1999.

28. N.P. Torrington-Smith, H.T. Mouftah and M.H. Rahman, "An evaluation of optical switch architectures utilizing wavelength converters," Electrical and Computer Eng., Canadian Conf., vol. 2 pp. 1008 -1013, 2000. 
29. R.A. Barry and P.A. Humblet, "Models of blocking probability in all-optical networks with an without wavelength changers, "IEEE J. Selected Areas in Communications, vol. 14, pp. 858-867, 1996.

30. R. Kannan, "The KR-Benes network: a control-optimal rearrangeable permutation network," IEEE Tran. on Computers, vol. 54, no. 5, pp. 534-544, 2005.

31. S. Subramaniam, M. Azizoglu, and A.K. Somani, "On optimal converter placement in wavelength-routed networks, "IEEE/ACM Tran. on Networking, vol. 7, pp. 754-766, 1999.

32. T.E. Stern and K. Bala. Multiwavelength optical networks: a layered approach. Addison Wesley, 1999.

33. T.T. Lee and S.Y. Liew, "Parallel routing algorithms in Benes-Close networks," IEEE Tran. on Communications, vol. 50, no. 11, pp. 1841-1847, November 2002.

34. V.E. Benes, "On rearrangeable three-stage connecting networks," Bell Syst. Tech. J., vol. XLI, no. 5, Sept. 1962.

35. W. J. Dally and B. Towles. Principles and practices of interconnection networks. Morgan Kaufmann Publishers, 2004.

36. X. Qin, and Y. Yang, "Nonblocking WDM switching networks with full and limted wavelegnth conversion," IEEE Transactions on Communications, vol. 50, no. 12, pp. 2032-2041, 2002.

37. Y. Yang, J. Wang, and C. Qiao, "Nonblocking WDM multicast switching networks," IEEE Tran. on Parallerl and distributed systems, vol. 11, no. 12, Dec. 2000, pp.1274-1287.

38. Y. Yang and J. Wang, "Desiging WDM optical interconnects with full connectivity by using limited wavelength conversion," IEEE Transactions on Computers, vol. 53, no. 12, pp. 1547-1556, 2004.

39. Y. Yang and J. Wang, "Cost-effective designs of WDM optical interconnects," IEEE Transactions on Parallel and Distributed Sys., vol. 16, no. 1., pp. 51-66, 2005. 